\title{
Integrated Cell Culture-Mass Spectrometry Method for Infectious Human
}

\section{Virus Monitoring}

5 Yinyin Ye, $\uparrow$ Linbo Zhao, + Michael J. Imperiale, + Krista R. Wigginton*,$\uparrow$

$7 \nmid$ Department of Civil and Environmental Engineering, University of Michigan, Ann Arbor,

8 Michigan 48109, United States

$9 \ddagger$ Department of Microbiology and Immunology, University of Michigan, Ann Arbor, Michigan

10 48109, United States

11

$12 *$ Corresponding author

13 Mailing address: Department of Civil and Environmental Engineering, 1351 Beal Ave., 181

14 EWRE, Ann Arbor, MI 48109-2125, USA. Phone: +1 (734) 763-2125; Fax: +1 (734) 764-4292;

15 E-mail: kwigg@umich.edu

16

17

18

19

20

21

22 
L2 and DBT cell lines. L2 and DBT cells (Table S1) were grown in Dulbecco's Modified

24 Eagle Medium (DMEM, Life Technologies 11960) supplemented with 10\% newborn bovine

25 serum (Life Technologies), $2 \mathrm{mM}$ of L-glutamine (Life Technologies), 100 units/mL of penicillin

26 and $100 \mu \mathrm{g} / \mathrm{mL}$ of streptomycin (Life Technologies) at $37{ }^{\circ} \mathrm{C}$ and $5 \% \mathrm{CO}_{2}$.

Vero and BSC-1 cell lines. Vero and BSC-1 cells (Table S1) were grown in DMEM (Life

29 Technologies 12430 ) with $10 \%$ newborn bovine serum, 100 units $/ \mathrm{mL}$ of penicillin and $100 \mu \mathrm{g} / \mathrm{mL}$

30 of streptomycin at $37^{\circ} \mathrm{C}$ and $5 \% \mathrm{CO}_{2}$.

BGMK cell lines. BGMK cells (Table S1) were cultured in Minimum Essential Medium (MEM, Sigma M2279) supplemented with 10\% fetal bovine serum (Sigma), 2 mM of L-glutamine,

$34100 \mathrm{units} / \mathrm{mL}$ of penicillin and $100 \mu \mathrm{g} / \mathrm{mL}$ streptomycin, $0.25 \mu \mathrm{g} / \mathrm{mL}$ of Amphotericin, $50 \mu \mathrm{g} / \mathrm{mL}$

35 of Kanamycin sulfate (Corning), $1 \mathrm{mM}$ of sodium pyruvate (Corning), $1 \times$ of NEAA (Life 36 Technologies), and $20 \mathrm{mM}$ of HEPES buffer (Lonza).

MHV preparation and plaque assay. Murine hepatitis virus (MHV) strain A59 propagation

39 (in DBT cells) and enumeration with plaque assays (in L2 cells) were based on a previously 40 published protocol. ${ }^{1}$ The propagated MHV stock $\left(\sim 10^{6} \mathrm{PFU} / \mathrm{mL}\right)$ was filtered through a $0.22 \mu \mathrm{m}$ 41 PES membrane and stored at $-80{ }^{\circ} \mathrm{C}$.

BKPyV preparation. Human BK polyomavirus (BKPyV) Dunlop variant was propagated in $44293 \mathrm{TT}$ cells according to a previously published protocol. ${ }^{2}$ Briefly, BK polyomavirus was added 45 to $293 \mathrm{TT}$ cell lines of 70-80\% confluency at a MOI of 0.1 infectious units per cell. After $1 \mathrm{~h}$ at 4 
${ }^{\circ} \mathrm{C}$ the infection media was removed and replaced with fresh media containing $10 \%$ serum. Cells

47 were incubated at $37{ }^{\circ} \mathrm{C}$ and $5 \% \mathrm{CO}_{2}$ for 10 days. The propagated BKPyV stock $\left(\sim 10^{8}\right.$ Infectious units/mL) was purified by $\mathrm{CsCl}$ gradient, dialyzed, and stored at $-20{ }^{\circ} \mathrm{C}$.

Infection. Cell lines were grown to $\sim 80-90 \%$ confluency in culture plates, washed with ice-

51 cold phosphate-buffered saline (PBS, Life Technologies 10010), and inoculated with virus-

52 containing samples. The inoculum and cells were incubated for $1 \mathrm{~h}$ at $37^{\circ} \mathrm{C}$ with manual rocking

53 every $15 \mathrm{~min}$. At this point, the inoculum was removed, and cells were sustained with their 54 respective culture media containing $2 \%$ serum.

LC gradient and MS instrument settings. A $20 \mu \mathrm{L}$ aliquot of the digested sample was loaded to a Dionex UltiMate 3000 LC system (ThermoFisher Scientific) equipped with an Accucore aQ column $(50 \mathrm{~nm} \times 2.1 \mathrm{~mm}, 2.6 \mu \mathrm{m}$ particle size, ThermoFisher Scientific) and an Accucore aQ

59 Defender guard column (ThermoFisher Scientific) at a flow rate of $200 \mu \mathrm{L} / \mathrm{min}$. Solvent A 60 consisted of Milli-Q water with $0.1 \%$ formic acid, and solvent B consisted of LC-MS grade 61 acetonitrile with $0.1 \%$ formic acid. The solvent gradient began at $94 \%$ solvent $\mathrm{A}$ and $6 \%$ solvent 62 B for 3 min. Solvent B increased linearly to $40 \%$ over $30 \mathrm{~min}$ and then to $80 \%$ over 2 min, and 63 was then maintained at $80 \%$ B for 5 min. Solvent B was then decreased to $6 \%$ where it remained 64 for 5 min to equilibrate the column. The LC-separated sample was analyzed by a Q Exactive 65 Orbitrap high-resolution mass spectrometer (ThermoFisher Scientific) in positive ion-mode 66 Capillary temperature of electrospray was set at $300^{\circ} \mathrm{C}$. For the MS scans, resolution was set as 67 70,000, and automatic gain control (AGC) target was 5e5. For the MS/MS, the top 20 ions were 68 selected for fragmentation with an isolation window of 1.6 Da. The resolution of MS/MS spectra 
69 was set as 35,000 , and the AGC target was 2e5. The normalized collision energy was 30 . The 70 dynamic exclusion was $20 \mathrm{sec}$.

71

72

73

74

75

76

77

78

79

80

81

82

83

84

85 


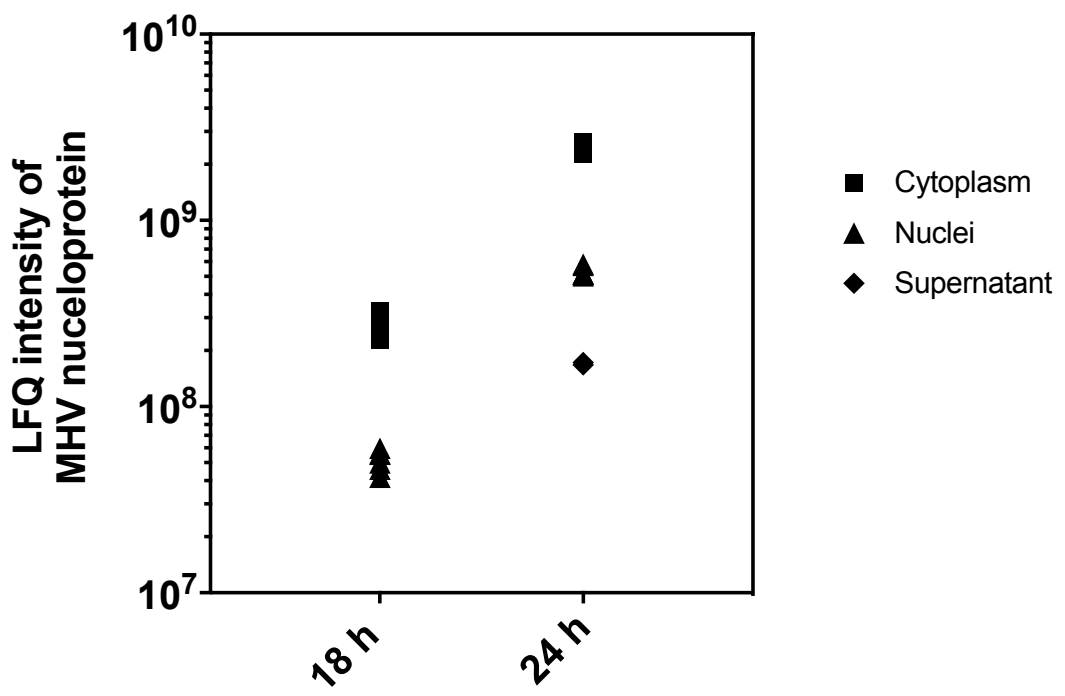

87 Figure S1. Label-free quantification (LFQ) intensity of MHV nucleoproteins in different protein 88 extracts measured by LC-MS/MS and quantified by LFQ in MaxQuant. Proteins were extracted

89 from culture media (supernatant), cell cytoplasm, and nuclei of MHV host L2 cells at 18 and 24

90 hours post infection. L2 cells $\left(\sim 23 \mathrm{~cm}^{2}\right.$ culture areas) were infected with 360 plaque forming units 91 (PFUs) of MHV.

92

93

94

95 


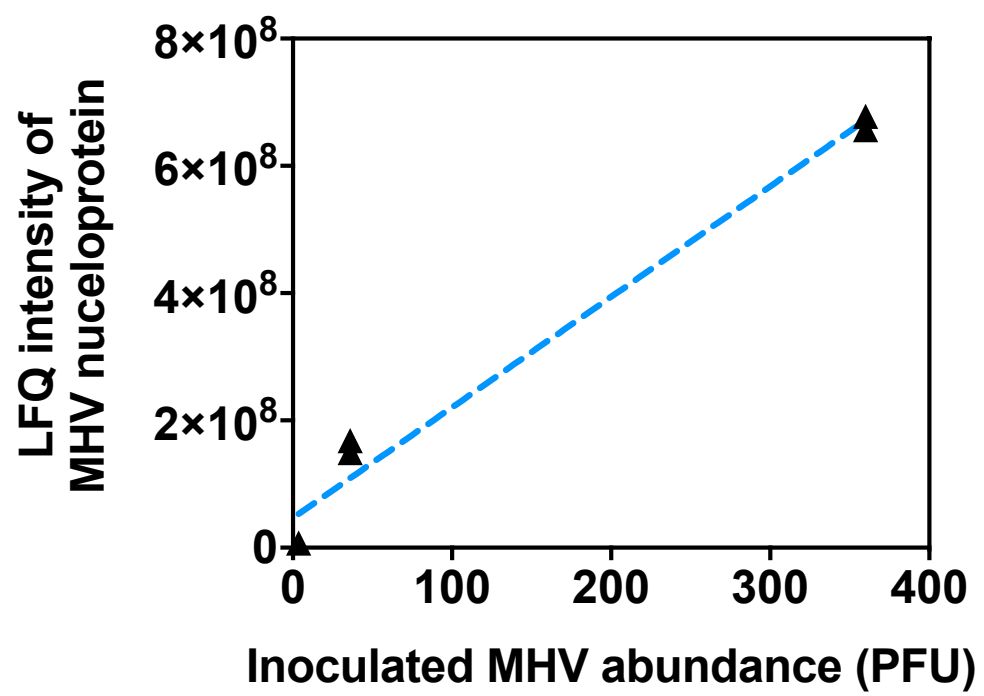

97 Figure S2. Correlation of MHV nucleoprotein LFQ intensity and MHV abundance in original

98 samples. MHV were suspended in media and 360 PFU, 36 PFU, 3.6 PFU were inoculated to the

99 L2 cells. Proteins were extracted from L2 cytoplasm at 27 to 30 hours post infection. Linear 100 regression result was LFQ intensity $=1.7 \times 10^{6} \cdot \mathrm{PFU}+4.7 \times 10^{7}\left(\mathrm{R}^{2}=0.98\right)$. 


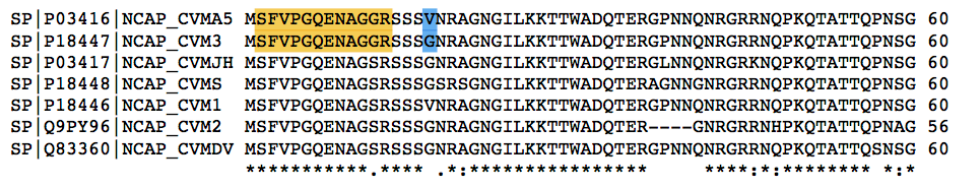

SP |P03416|NCAP_CVMA5 SVVPHYSWFSGITQFQKGKEFQFAEGQGVPIANGIPASEQKGYWYRHNRRSFKTPDGQQK 120 SP P18447 NCAP CVM3 SVVPHYSWFSGITOFQKGKEFQFAEGOGVPIANGIPASEOKGYWYRHNRRSFKTPDGQQK 120 SP P03417|NCAP_CVMJH SVVPHYSWFSGITQFQKGKEFQFAQGQGVPIANGIPASQQKGYWYRHNRRSFKTPDGQQK 120 SP P18448 NCAP CVMS SVVPHYSWFSGITQFQKGKEFQFVQGQGVPIANGIPASEQKGYWYRHNRRSFKTPDGQQK 120 SP P18446 NCAP_CVM1 SVVPHYSWFSGITQFQKGKEFQFAQGQGVPIANGIPASEQKGYWYRHNRRSFKTPDGQQK 120 SP Q9PY96 NCAP CVM2 SVVPHYSWF SGITQFQKGKEFQFAQGQGVPIASGIPASEQKGYWYRHNRRSFKTPDGQHK 116 SP $\mid$ Q83360|NCAP_CVMDV SVVPHYSWFSGITQFQKGKEFQFADGQGVPIANGIPASEQKGYWYRHNRRSFKTPDGQQK 120

SP|P03416|NCAP_CVMA5 QLLPRWYFYYLGTGPHAGASYGDSIEGVFWVANSQADTNTRSDIVERDPSSHEAIPTRFA 180 SP P18447 NCAP_CVM3 QLLPRWYFYYLGTGPHAGASYGDSIEGVFWVANSQADTNTRSDIVERDPSSHEAIPTRFA 180 SP P03417 NCAP_CVMJH QLLPRWYFYYLGTGPYAGAEYGDDIEGVVWVASQQAETRTSADIVERDPSSHEAIPTRFA 180 SP P18448 NCAP_CVMS QLLPRWYFYYLGTGPHAGAEYGDDIEGVVWVASQQADTKTTADIVERDPSSHEAIPTRFA 180 SP P18446| NCAP_CVM1 QLLPRWYFYYLGTGPHAGAEYGDDIDGVVWVASQQADTKTTADIVERDPSSHEAIPTRFA 180 SP Q9PY96 NCAP_CVM2 QLLPRWYFYYLGTGPHAGAEYGDDIEGVVWVASQQADTKTTADVVERDPSSHEAIPTRFA 176 SP $\mid$ Q83360|NCAP_CVMDV QLLPRWYFYYLGTGPHAGATYGDSIEGVFWVANSQADTNTRSDIVERDPSSHEAIPTRFA 180

SP|P03416|NCAP_CVMA5 PGTVLPQGFYVEGSGRSAPASRSGSRSQSRGPNNRARSSSNQRQPASTVKPDMAEEIAAL 240 SP P18447|NCAP_CVM3 PGTVLPQGFYVEGSGRSAPASRSGSRSQSRGPNNRARSSSNQRQPASTVKPDMAEEIAAL 240 SP | P03417|NCAP_CVMJH PGTVLPQGFYVEGSGRSAPASRSGSRPQSRGPNNRARSSSNQRQPASTVKPDMAEEIAAL 240 SP P18448|NCAP_CVMS PGTVLPQGFYVEGSGRSAPASRSGSRSQSRGPNNRARSSSNQRQPASTVKPDMAEEIAAL 240 SP 18446 |NCAP_CVM1 PGTVLPQGFYVEGSGRSAPASRSGSRSQSRGPNNRARSSSNQRQPASTVKPDMAEEIAAL 240 SP Q9PY96|NCAP_CVM2 PGTVLPQGFYVEGSGRSAPASRSGSRSQSRGPNNRARSSSNQRQPASAVKPDMAEEIAAL 236 SP $\mid$ Q83360|NCAP_CVMDV PGTVLPQGFYVEGSGRSAPASRSGSRSQSRGSNNRARSSSNQRQPASTVKPDMAEEIAAL 240

SP $\mid$ P03416|NCAP_CVMA5 VLAKLGKDAGQPKQVTKQSAKEVRQKILNKPRQKRTPNKQCPVQQCFGKRGPNQNFGGSE 300 SP P18447| NCAP_CVM3 VLAKLGKDAGQPKQVTKQSAKEVRQKILNKPRQKRTPNKQCPVQQCFGKRGPNQNFGGSE 300 SP P03417|NCAP_CVMJH VLAKLGKDAGQPKQVTKQSAKEVRQKILNKPRQKRTPNKQCPVQQCFGKRGPNQNFGGPE 300 SP P18448| NCAP_CVMS VLAKLGKDAGQPKQVTKQSAKEVRQKILNKPRQKRTPNKQCPVQQCFGKRGPNQNFGGSE 300 SP P18446 NCAP_CVM1 VLAKLGKDAGQPKQVTKQSAKEVRQKILNKPRQKRTPNKQCPVQQCFGKRGPNQNFGGSE 300 SP Q9PY96|NCAP_CVM2 VLAKLGKDAGQPKQVTKQSAKEVRQKILTKPRQKRTPNKQCPVQQCFGKRGPNQNFGGSE 296 SP $\mid$ Q83360|NCAP_CVMDV VLAKLGKDAGQPKQVTKQSAKEVRQKILNKPRQKRTPNKQCPVQQCFGKRGPNQNFGGSE 300

SP|P03416|NCAP_CVMA5 MLKLGTSDPQFPILAELAPTVGAFFFGSKLELVKKNSGGADEPTKDVYELQYSGAVRFDS 360 SP $\mid$ P18447 NCAP_CVM3 MLKLGTSDPQFPILAELAPTVGAFFFGSKLELVKKNSGGADEPTKDVYYELQYSGAVRFDS 360 SP P03417 NCAP_CVMJH MLKLGTSDPQFPILAELAPTAGAFFFGSKLELVKKNSGGADGPTKDVYELQYSGGAVRFDS 360 SP P18448 NCAP_CVMS MLKLGISDPQPPILAELAPIAGAFFFGSKLELVKKNSGGADEPTKDVYELQYSGAVRFDS 360 SP P18446 NCAPCVM1 MLKLGISDPQFPILAELAPTSAFFFSKLELVKKNSGGADDPIKDVYLLQYSGAIRFDS 360 SP Q9PY96|NCAP_CVM2 MLKLGTSDPQFPILAELAPTPSAFFFGKLELVKKNSGGADEPTKDVYELQYSGAIRFDS 356 SP $|Q 83360|$ NCAP_CVMDV MLKLGTSDPQFPILAELAPTPGAFFFGSLELVKKNSGGVDEPTKDVYELLYSGAIRFDS 360

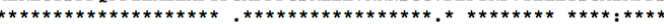

SP $\mid$ P03416|NCAP_CVMA5 TLPGFETIMKVLNENLNAYQK-DGGADVVSPKPQRKGRRQAQEKKDEVDNVSVAKPKSSV 419 SP P18447|NCAP CVM3 TLPGFETIMKVLNENLNAYOK-DGGADVVSPKPORKGRROAOEKKDEVDNVSVAKPKSSV 419 SP $\mid$ P18447 $\mid$ NCAP_CVM3 TLPGFETIMKVLNENLNAYQK-DGGADVVSPKPQRKGRRQAQEKKDEVDNVSVAKPKSSV 419

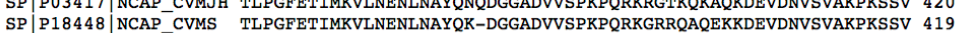

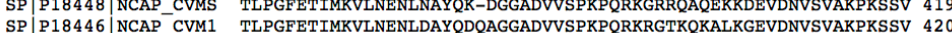
SP P18446 | NCAP_CVM2 TLPGFETIMKVLTENLNAYQDQAGSVDLVSPKPPRRGRRQAQEKKDEVDNVSVAKPKSLV 416 SP | $83360 \mid$ NCAP CVMDV TLPGFETIMKVLNENLNAYQNQAGGVDVVSPKPQRKGRRQAQEKKDEVDNVSVAKPKSSV 420

SP|P03416|NCAP_CVMA5 QRNVSRELTPEDRSLLAQILDDGVVPDGLEDDSNV 454 SP P18447 NCAP CVM3 ORNVSRELTPEDRSLLAOILDDGVVPDGLEDDSNV 45 SP P03417 SP P18448 $\mid$ NCAP CVMS ORNVSRELTPEDRSLLAQILDDGVVPDGLEDDSNV 454 SP P18446| NCAP CVM1 QRNVSRELTPEDRSLLAQILDDGVVPDGLEDDSNV 455 SP P 18446 T SP $183360 \mid$ NCAP CVMDV RRVSPETPDSLA

102 Figure S3. Multiple sequence alignment of MHV nucleoproteins for seven MHV strains available

103 in the Uniprot database, including MHV-A59 (P03146), MHV-3 (P18447), MHV-JHM (P03417),

104 MHV-2 (Q9PY96), MHV-1 (P18446), and MHV-S (P18448), and MHV-DVIM (Q83360). The

105 strain 3- and A59-specific peptides detected by LC-MS/MS were highlighted in yellow. The

106 distinct amino acid residues of strain 3 and strain A59 nucleoproteins were highlighted in blue. 


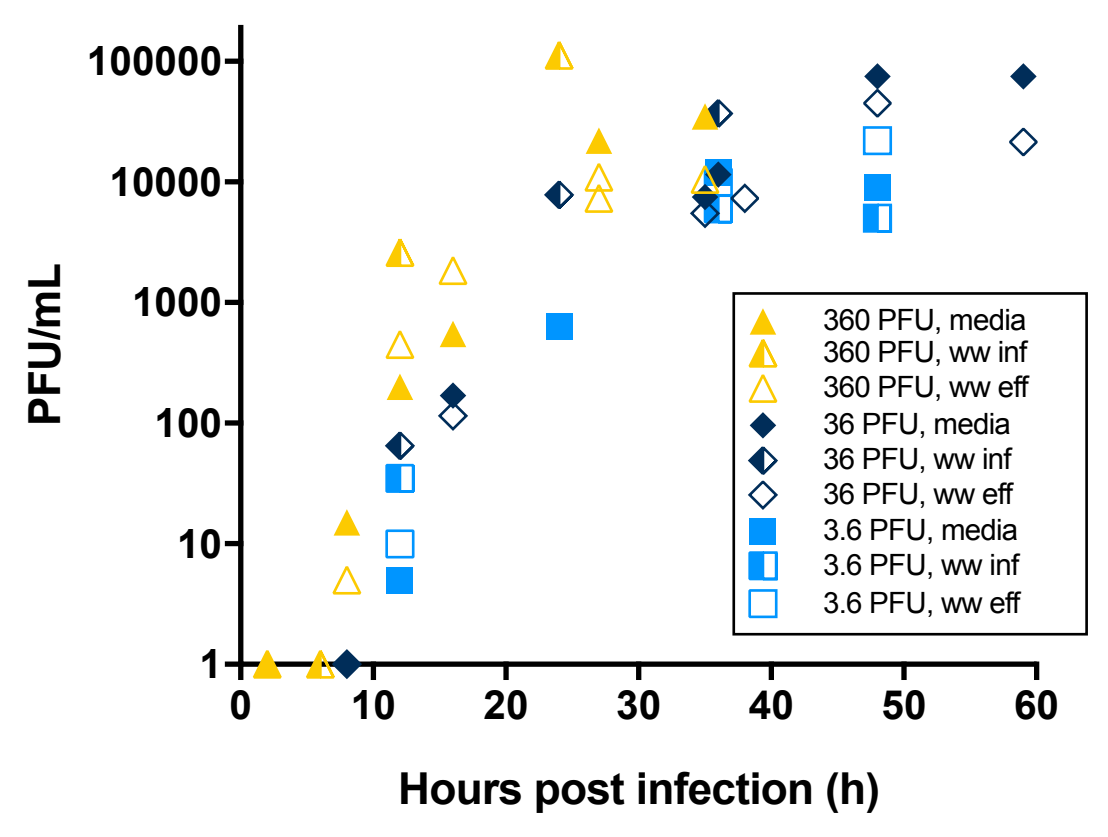

108 Figure S4. MHV propagation curves. L2 cell lines inoculated with MHV suspended in various

109 aqueous environments, including culture media (media), concentrated primary influent (INF), and

110 concentrated UV-treated effluent (EFF).

111

112

113

114 


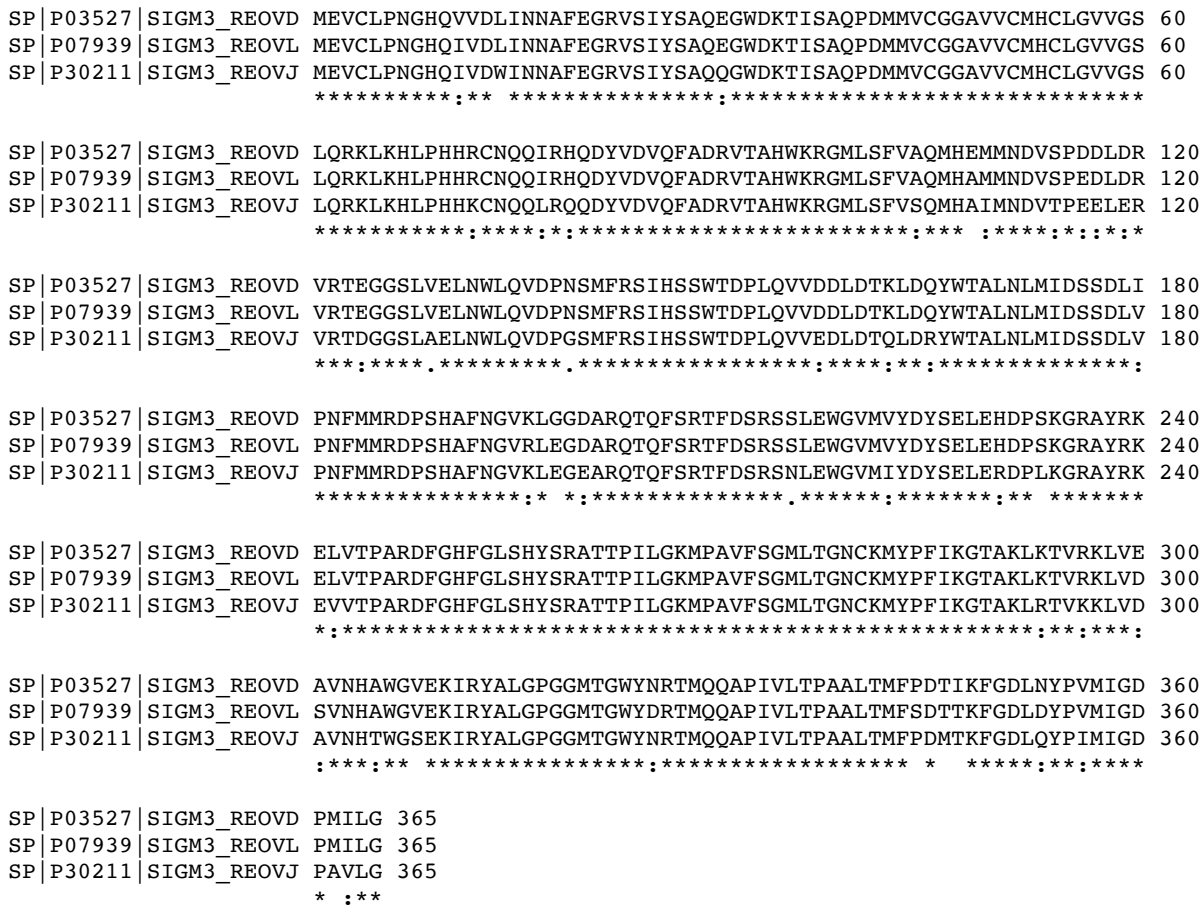

116 Figure S5. Multiple sequence alignment of reovirus $\sigma 3$ proteins. Sequence similarity: $88.9 \%$.

117 Proteins include $\sigma 3$ of reovirus type 1 (P07939), reovirus type 2 (P30211), and reovirus type 3

118 (P03527). 
SP|P11078|MU1_REOVD MGNASSIVQTINVTGDGNVFKPSAETSSTAVPSLSLSPGMLNPGGVPWIAVGDETSVTSP 60 SP|P11077|MU1_REOVL MGNASSIVQTINVTGDGNVFKPSAETSSTAVPSLSLSPGMLNPGGVPWIAIGDETSVTSP 60 SP|P12397|MU1_REOVJ MGNASSIVQTINVTGDGNVFKPSAETSSTAVPSLSLSPGMLNPGGVPWIAIGDETSVTSP 60 $* * * * * * * * * * * * * * * * * * * * * * * * * * * * * * * * * * * * * * * * * * * * * * * * * *: * * * * * * * * *$

SP |P11078|MU1_REOVD GALRRMTSKDIPETAIINTDNSSGAVPSESALVPYIDEPLVVVTEHAITNFTKAEMALEF 120 SP $\mid$ P11077|MU1 REOVL GALRRMTSKDIPETAIINTDNSSGAVPSESALVPYNDEPLVVVTEHAIANFTKAEMALEF 120 SP |P12397|MU1 REOVJ GALRRMTSKDIPETAIINTDNSSGAVPSESALVPYNDEPLVVVTEHAIANFTKAEMALEF 120 $* * * * * * * * * * * * * * * * * * * * * * * * * * * * * * * * * * * * * * * * * * * * * *: * * * * * * * * * * *$

SP |P11078|MU1 REOVD NREFLDKMRVLSVSPKYSDLLTYVDCYVGVSAROALNNFOKOVPVITPTROTMYVDSIOA 180 SP $\mid$ P11077|MU1 REOVL NREFLDKLRVLSVSPKYSDLLTYVDCYVGVSAROALNNFOKOVPVITPTROTMYVDSIOA 180 SP|P12397|MU1_REOVJ NREFLDKLRVLSVSPKYSDLLTYVDCYVGVSARQALNNFQKQVPVITPTRQTMYVDSIQA 180

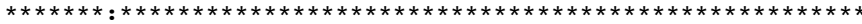

SP |P11078|MU1 REOVD ALKALEKWEIDLRVAOTLLPTNVPIGEVSCPMOSVVKLLDDOLPDDSLIRRYPKEAAVAL 240 SP $|\mathrm{P} 11077| \mathrm{MU} 1$ REOVL ALKALEKWEIDLRVAQTLLPTNVPIGEVSCPMQSVVKLLDDQLPDDSLIRRYPKEAAVAL 240 SP|P12397|MU1 REOVJ ALKALEKWEIDLRVAOTLLPTNVPIGEVSCPMOSVVKLLDDOLPDDSLIRRYPKEAAVAL 240 $* * * * * * * * * * * * * * * * * * * * * * * * * * * * * * * * * * * * * * * * * * * * * * * * * * * * * * * * * * * *$

SP |P11078|MU1_REOVD AKRNGGIQWMDVSEGTVMNEAVNAVAASALAPSASAPPLEEKSKLTEQAMDLVTAAEPEI 300 SP $\mid$ P1 1077 MU1 REOVL AKRNGGIOWMDVSEGTVMNEAVNAVAASALAPSASAPPLEEKSKLTEOAMDLVTAAEPEI 300 SP |P12397|MU1_REOVJ AKRNGGIQWMDVSEGTVMNEAVNAVAASALAPSASAPPLEEKSKLTEQAMDLVTAAEPEI 300 $* * * * * * * * * * * * * * * * * * * * * * * * * * * * * * * * * * * * * * * * * * * * * * * * * * * * * * * * * * * *$

SP |P11078|MU1 REOVD IASLAPVPAPVFAIPPKPADYNVRTLRIDEATWLRMIPKSMNTPFOIOVTDNTGTNWHLN 360 SP $|\mathrm{P} 11077| \mathrm{MU1}$ 1_REOVL IASLVPVPAPVFAIPPKPADYNVRTLKIDEATWLRMIPKTMGTPFQIQVTDNTGTNWHLN 360 SP |P12397|MU1_REOVJ IASLVPVPAPVFAIPPKPADYNVRTLKIDEATWLRMIPKTMNTPFQIQVTDNTGTSWHMN 360

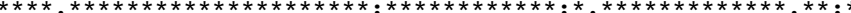

SP|P11078|MU1_REOVD LRGGTRVVNLDQIAPMRFVLDLGGKSYKETSWDPNGKKVGFIVFQSKIPFELWTAASQIG 420 SP $|P 11077|$ MU1_REOVL LRGGTRVVNLDQIAPMRFVLDLGGKSYKETSWDPNGKKVGFIVFQSKIPFELWTAASQIG 420 SP $\mid$ P12397 1 MU1 REOVJ LRGGTRVVNLDQIAPMRFVLDLGGKSYKETSWDPNGKKVGFIVFQSKIPFELWTAASQIG 420 $* * * * * * * * * * * * * * * * * * * * * * * * * * * * * * * * * * * * * * * * * * * * * * * * * * * * * * * * * * * *$

SP |P11078|MU1 REOVD OATVVNYVOLYAEDSSFTAOSIIATTSLAYNYEPEOLNKTDPEMNYYLLATFIDSAAITP 480 SP $\mid$ P11077|MU1 REOVL OATVVNYVQLYAEDSSFTAOSIIATTSLAYNYEPEOLNKTDPEMNYYLLATFIDSAAITP 480 SP|P12397|MU1_REOVJ QATVVNYVQLYAEDSSFTAQSIIATTSLAYNYEPEQLNKTDPEMNYYLLAAFIDSAAIST 480

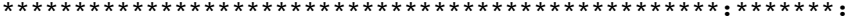

SP |P11078|MU1 REOVD TNMTOPDVWDALLTMSPLSAGEVTVKGAVVSEVVPADLIGSYTPESLNASLPNDAARCMI 540 SP |P11077|MU1_REOVL TNMTQPDVWDALLTMSPLSAGEVTVKGAVVSEVVPAELIGSYTPESLNASLPNDAARCMI 540 SP $|\mathrm{P} 12397|$ MU1_REOVJ SNMTQPDVWDALLTMSPLSAGEVTVKGAVVSEVIPADLVGSYTPESLNASLPNDAARCMI 540 $: * * * * * * * * * * * * * * * * * * * * * * * * * * * * * * * *: * *: *: * * * * * * * * * * * * * * * * * * * * *$

SP|P11078|MU1_REOVD DRASKIAEAIKIDDDAGPDEYSPNSVPIQGQLAISQLETGYGVRIFNPKGILSKIASRAM 600 SP $\mid$ P11077|MU1 REOVL DRASKIAEAIKIDDDAGPDEYSPNSVPIQGQLAISQLETGYGVRIFNPKGILSKIASRAM 600 SP |P12397|MU1_REOVJ DRASKIAEAIKIDDDAGPDEYSPNSVPIQGQLAISOLETGYGVRIFNPKGILSKIASRAM 600 $* * * * * * * * * * * * * * * * * * * * * * * * * * * * * * * * * * * * * * * * * * * * * * * * * * * * * * * * * * * *$

SP |P11078|MU1 REOVD OAFIGDPSTIITOAAPVLSDKNNWIALAOGVKTSLRTKSLSAGVKTAVSKLSSSESIONW 660 SP |P11077|MU1 REOVL OAFIGDPSTIITOAAPVLSDKNNWIALAOGVKTSLRTKSLSAGVKTAVSKLSSSESIQNW 660 SP |P12397|MU1_REOVJ QAFIGDPSTIITQAAPVLSDKNNWIALAQGVKTSLRTKSLSAGVKTAVSKLSSSESIQSW 660

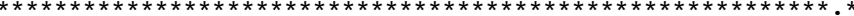

SP $\mid$ P11078|MU1 REOVD TQGFLDKVSAHFPAPKPDCPTSGDSGESSNRRVKRDSYAGVVKRGYTR 708 SP|P11077|MU1_REOVL TQGFLDKVSTHFPAPKPDCPTNGDGSEPSARRVKRDSYAGVVKRGYTR 708 SP |P12397|MU1 REOVJ TOGFLDKVSTHFPAPKPDCPOSGDSGDGSARRLKRDSYAGVVKRGYTR 708

122 Figure S6. Multiple sequence alignment of human reovirus $\mu 1$ proteins. Sequence similarity:

123 96.2\%. Proteins include $\mu 1$ of reovirus type 1 (P11077), reovirus type 2 (P12397), and reovirus

124 type 3 (P11078). 
128 Table S1. Cell lines and viruses used in this study.

\begin{tabular}{|c|c|c|c|c|c|}
\hline Cell line & Organism & Tissue; Morphology & Source & $\begin{array}{l}\text { Example viruses that can } \\
\text { be cultured }\end{array}$ & Citations \\
\hline L2 & Mus musculus & lung; epithelial & \multirow{2}{*}{$\begin{array}{l}\text { Dr. Julian Leibowitz's lab at } \\
\text { Texas A\&M Health Science } \\
\text { Center College of Medicine }\end{array}$} & Murine hepatitis virus & \\
\hline DBT & Mus musculus & lung; epithelial & & Murine hepatitis virus & \\
\hline RPTE & Homo sapiens & $\begin{array}{l}\text { Renal cortex, } \\
\text { proximal tubules, } \\
\text { epithelium; epithelial- } \\
\text { like }\end{array}$ & \multirow{3}{*}{$\begin{array}{l}\text { Dr. Michael J. Imperiale's lab, } \\
\text { University of Michigan }\end{array}$} & BK polyomavirus & \\
\hline Vero & $\begin{array}{l}\text { Cercopithecus } \\
\text { aethiops }\end{array}$ & kidney; epithelial & & $\begin{array}{l}\text { Poliovirus, coxsackie } \\
\text { virus, echovirus, } \\
\text { reovirus, picornavirus } \\
\text { simian virus } 40 \\
\end{array}$ & 3,4 \\
\hline BSC-1 & $\begin{array}{l}\text { Cercopithecus } \\
\text { aethiops }\end{array}$ & kidney; epithelial & & $\begin{array}{l}\text { Poliovirus, coxsackie } \\
\text { virus, echovirus, } \\
\text { reovirus, hepatitis A } \\
\text { virus, simian virus } 40 \\
\end{array}$ & $3,5,6$ \\
\hline BGMK & $\begin{array}{l}\text { Cercopithecus } \\
\text { aethiops }\end{array}$ & kidney; epithelial & Dr. Tamar Kohn's lab, EPFL & $\begin{array}{l}\text { Reovirus, poliovirus, } \\
\text { coxsackie virus, } \\
\text { echoviruses, adenovirus }\end{array}$ & 7,8 \\
\hline Virus & & & Source & & \\
\hline \multicolumn{3}{|c|}{ Murine hepatitis virus strain A59 } & \multicolumn{3}{|c|}{$\begin{array}{l}\text { Dr. Julian Leibowitz's lab at Texas A\&M Health Science } \\
\text { Center College of Medicine }\end{array}$} \\
\hline \multicolumn{3}{|c|}{ Human BK polyomavirus Dunlop variant } & Dr. Michael J. Imperiale's lab, & iversity of Michigan & \\
\hline
\end{tabular}

132 Table S2-S5 were provided in a separate XLSX file:

133 Table S2. Viral peptides detected by LC-MS/MS in cytoplasm protein extracts of L2 cells. L2 cell

134 lines were infected by MHV suspended in culture media at $360 \mathrm{PFU}, 36 \mathrm{PFU}$, and 3.6 PFU.

135 Proteins were extracted from the L2 cytoplasm at indicated hours post infection. Strain-specific 136 peptides of MHV- A59 and MHV-3 were shown in red.

138 Table S3. Viral peptides detected by LC-MS/MS in nuclear protein extracts of human renal 139 proximal tubule epithelial (RPTE) cells. RPTE cell lines were infected by BK polyomavirus 140 Dunlop variant at an infectious virus-to-cell ratio of 0.1. Proteins were extracted from the nuclei 141 of RPTEC at 3 days post infection. 
143 Table S4. Viral peptides detected by LC-MS/MS in cytoplasm protein extracts of L2 cells. L2

144 cells were infected by MHV suspended in wastewater primary influent concentrates (INF) and

145 post-UV effluent concentrates (EFF) at 360 PFU, 36 PFU and 3.6 PFU. Proteins were extracted

146 from the cytoplasm of L2 cells at indicated hours post infection. Strain-specific peptides of

147 MHV- A59 and MHV-3 were shown in red.

149 Table S5. Viral peptides detected by LC-MS/MS in cytoplasm protein extracts of Vero, BSC-1

150 and BGMK cells. These cell lines were infected with wastewater samples, including primary

151 influent concentrates (INF), secondary effluent (Pre-UV EFF), and UV-treated effluent (Post-UV

152 EFF). Proteins were extracted from the cell cytoplasm at 14 days post infection for Vero and BSC-

1531 and at 10 days post infection for BGMK. Detected peptides in reovirus type 1 proteins were

154 indicated by grey squares, in type 2 proteins were indicated by red squares, and in type 3 proteins

155 were indicated by green squares.

157 Table S6. Proteins expressed during reovirus replication.

\begin{tabular}{|l|l|l|}
\hline Location & Protein & $\begin{array}{l}\text { Molecule copies in } \\
\text { reovirus virion }\end{array}$ \\
\hline \multirow{4}{*}{$\begin{array}{l}\text { Structural proteins } \\
\text { Outer capsid }\end{array}$} & $\mu 1$ & 600 \\
\cline { 2 - 3 } & $\sigma 3$ & 600 \\
\cline { 2 - 3 } & $\lambda 2$ & 60 \\
\cline { 2 - 3 } & $\sigma 1$ & $36-48$ \\
\hline \multirow{3}{*}{$\begin{array}{l}\text { Structural proteins } \\
\text { Inner capsid }\end{array}$} & $\sigma 2$ & 150 \\
\cline { 2 - 3 } & $\lambda 1$ & 120 \\
\cline { 2 - 3 } & $\lambda 3$ & 12 \\
\cline { 2 - 3 } & $\mu 2$ & 12 \\
\hline \multirow{2}{*}{$\begin{array}{l}\text { Non-structural } \\
\text { proteins }\end{array}$} & $\sigma \mathrm{NS}$ & \\
\cline { 2 - 3 } & $\mu \mathrm{NS}$ & \\
\cline { 2 - 3 } & $\sigma 1 \mathrm{~s}$ & \\
\hline
\end{tabular}


References:

161

162

163

164

165

166

167

168

169

170

171

172

173

174

175

176

177

178

179

180

181

182

183

184

185

186

187

188

189

190

191

(1) Leibowitz, J.; Kaufman, G.; Liu, P. Coronaviruses: propagation, quantification, storage, and construction of recombinant mouse hepatitis virus. Curr. Protoc. Microbiol 2011, 21 (15E.1), 1-52.

(2) Mengxi Jiang, Johanna R. Abend, Billy Tsai, Michael J. Imperiale. Early Events during BK Virus Entry and Disassembly. J. Virology 2009, 83 (3) 1350-1358

(3) Payment, P.; Ayache, R.; Trudel, M. A survey of enteric viruses in domestic sewage. Can. J. Microbiol. 2011, 29 (1), 111-119.

(4) Gelb, L. D.; Kohne, D. E.; Martin, M. A. Quantitation of simian virus 40 sequences in African green monkey, mouse and virus-transformed cell genomes. J. Mol. Biol. 1971, 57 (1), 129-145.

(5) Gerba, C. P.; Sobsey, M. D.; Wallis, C.; Melnick, J. L. Adsorption of poliovirus onto activated carbon in wastewater. J. Chem. Eng. 1975, 9 (8), 727-731.

(6) Sompayrac, L. M.; Danna, K. J. Efficient infection of monkey cells with DNA of simian virus 40. Proc. Natl. Acad. Sci. 1981, 78 (12), 7575-7578.

(7) Schmidt, N. J.; Ho, H. H.; Riggs, J. L.; Lennette, E. H. Comparative sensitivity of various cell culture systems for isolation of viruses from wastewater and fecal samples. Appl. Environ. Microbiol. 1978, 36 (3), 480-486.

(8) Chapron, C. D.; Ballester, N. A.; Ballester, N. A.; Fontaine, J. H.; Fontaine, J. H.; Frades, C. N.; Frades, C. N.; Margolin, A. B.; Margolin, A. B. Detection of astroviruses, enteroviruses, and adenovirus types 40 and 41 in surface waters collected and evaluated by the information collection rule and an integrated cell culture-nested PCR procedure. Appl. Environ. Microbiol. 2000, 66 (6), 2520-2525.

(9) Dryden, K. A.; Wang, G.; Yeager, M.; Nibert, M. L.; Coombs, K. M.; Furlong, D. B.; Fields, B. N.; Baker, T. S. Early steps in reovirus infection are associated with dramatic changes in supramolecular structure and protein conformation: analysis of virions and subviral particles by cryoelectron microscopy and image reconstruction. J. Cell Biol. 1993, 122 (5), 1023-1041. 